\title{
Relative Spectral Difference Occurrence Matrix: A Metrologi- cal Spectral-Spatial Feature for Hyperspectral Texture Analysis
}

\author{
Rui Jian Chu' ${ }^{1}$, Noël Richard ${ }^{1}$, Christine Fernandez-Maloigne ${ }^{1}$, Jon Yngve Hardeberg ${ }^{2}$ \\ 1. XLIM Laboratory, JRU CNRS 7252, University of Poitiers, France \\ 2. Department of Computer Science, Norwegian University of Science and Technology, Norway
}

\begin{abstract}
We develop a spectral-spatial feature, Relative Spectral Difference Occurrence Matrix (RSDOM) for hyperspectral texture recognition. Inspired by Julesz's conjecture, the proposed feature is based on spectral difference approach and respects the metrological constraints. It simultaneously considers the distribution of spectra and their spatial arrangement in the hyperspectral image. The feature is generic and adapted for any number of spectral bands and range. We validate our proposition by applying a classification scheme on the HyTexiLa database. An accuracy comparable to local binary pattern approach is obtained, but at a much reduced cost due to the extremely small feature size.
\end{abstract}

\section{Introduction}

Hyperspectral imaging (HSI) has become increasingly popular with the advance of sensor technology and computational speed. Due to the ability of HSI in capturing the entire electromagnetic spectrum at hundreds of contiguous, narrow spectral bands, it allows non-destructive assessment of material with far superior discrimination. As such, hyperspectral texture analysis has received considerably attention and its application can be found in many fields including biomedical, industrial and remote sensing. For instances, hyperspectral texture analysis have been used in classification of biopsy samples [1,2], food quality inspection $[3,4]$ and ground classification of aerial imagery $[5,6]$.

Many assessment of hyperspectral texture are the direct outgrowth of approaches originally adapted for monospectral (intensity) images $[7,8,9]$. Facing the complexity of hyperspectral data, the common strategy is band selection $[10,11,12]$ or dimensionality reduction $[13,14,15]$ using Principal Component Analysis (PCA), Non-Negative Matrix Factorization (NMF) and others. Then, the multivariate texture assessment is performed in a marginal way (band by band) or cross-channel processing [16]. Such approach is data dependent and consequently, results from different dataset are not incomparable. Besides, the act of feature transformation in dimensionality reduction could corrupt a part of the physical content of the dataset [17]. For metrological purposes, all physical content need to be preserved so that the processing accuracy, uncertainty, and bias are quantifiable [18].

In this work, we attempt to develop a spectral-spatial feature for hyperspectral texture analysis. We begin by considering the Julesz conjecture, which states that human discrimination is possible between textures differing in low order statistics $[19,20]$. We next take inspiration from the pioneering work of Haralick and Ojala in texture analysis, namely Co-occurrence matrix [21] and Local Binary Pattern (LBP) [22]. We remark that the texture description using Co-occurrence matrix is complete, but its reduction into moments for similarity measure causes information losses. For LBP, the binarization provides a weak characterization of texture, but its similarity measure is extremely efficient. Following the work of [23] which relates Co-occurrence matrix

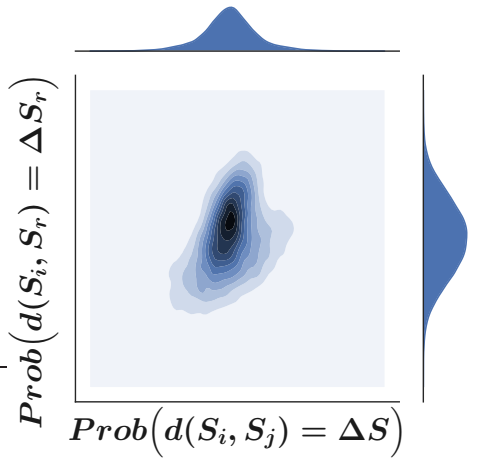

Figure 1: An illustration of RSDOM, combining spectral and spatial features

and histogram of differences, we combine the strength of both approaches and adapt it for HSI processing while respecting the metrological constraints. For enhanced discrimination, we incorporate the spectral feature in the texture assessment, thus inducing a spectral-spatial feature.

The rest of the article is organized in the following manner. We next describe our proposed spectral-spatial feature as well as its similarity measure. Then, we propose an experiment to test the feature with analysis and discussion, along with suggestions for future work. Finally, we present the conclusion for this work.

\section{Proposed method}

We are interested in a spectral-spatial feature for hyperspectral texture recognition that has the following properties: (I) computationally efficient; adapted for any spectral count and range, (II) metrological; conform to the science of measurement, and (III) compact; for concise representation. In the following, we describe the formulation of the feature in terms of its expected properties as well as its measure of similarity.

\section{A spectral-spatial feature}

We consider the description of hyperspectral texture from both spatial and spectral perspectives. The spatial feature examines the spatial arrangement in the image that gives rise to ideas such as smoothness, coarseness and pattern repeatability [24]. On the other hand, the spectral feature assesses the spectral information in the image that induces concepts such as intensity, saturation and purity. When combined, the spectral-spatial features provide a complete description of hyperspectral texture.

We begin by first considering the formulation of spatial feature $M_{s p a}$. Following Julesz's conjecture on texture discrimination based on low order statistics $[19,20]$, we describe the spatial arrangement in a hyperspectral image $H$ via the distribution of spectral difference between pixel pairs separated by a spatial vector $\vec{v}$. Suppose that $\vec{v}$ is specified by distance $d$ and direction 
$\theta, M_{\text {spa. }}$ is expressed as a probability density function given by:

$$
\begin{gathered}
M_{\text {spa. }}^{(d, \theta)}(\Delta S)=\operatorname{Prob}\left(d\left(S_{i}, S_{j}\right)=\Delta S\right), \forall i, j \in H, \\
\|\overrightarrow{i j}\|=d, \angle \overrightarrow{i j}=\theta
\end{gathered}
$$

where $d\left(S_{i}, S_{j}\right)=\Delta S$ is the spectral difference between spectra $S_{i}$ and $S_{j}$ of pixels $i$ and $j$ respectively, separated by $\vec{v}$.

Next, we consider the formulation of spectral feature $M_{\text {spe. }}$. We characterize the spectral variation in $H$ via spectral difference $d\left(S_{i}, S_{r}\right)$, where $i \in H$ and $S_{r}$ is a chosen reference. Doing so, we are able to reduce the description from a $P$-dimensional spectral space to a $p$-dimensional difference space, where $p \ll P$. Expressed as a probability density function, $M_{s p e}$. is given by:

$$
M_{\text {spe. }}\left(\Delta S_{r}\right)=\operatorname{Prob}\left(d\left(S_{i}, S_{r}\right)=\Delta S_{r}\right), \forall i \in H
$$

where $d\left(S_{i}, S_{r}\right)=\Delta S_{r}$ is the spectral difference between each pixel in the image and the reference. The choice of $S_{r}$ is made such that it lies just outside the convex hull of the spectral distribution [25] of the entire image set. This is to maximize discrimination between the textured images.

Combining $M_{\text {spa }}$ and $M_{\text {spe. }}$, we obtain the spectral-spatial feature $M$ as joint probability density function given by:

$$
\begin{gathered}
M^{(d, \theta)}\left(\Delta S, \Delta S_{r}\right)=\operatorname{Prob}\left(d\left(S_{i}, S_{j}\right)=\Delta S, d\left(S_{i}, S_{r}\right)=\Delta S_{r}\right), \\
\forall i, j \in H,\|\overrightarrow{i j}\|=d, \angle \overrightarrow{i j}=\theta
\end{gathered}
$$

termed as Relative Spectral Difference Occurrence Matrix (RSDOM). An illustration of RSDOM is shown in Figure 1.

To explain further on the choice of $S_{r}$, consider the working example in Figure 2. Suppose there are two textured images (each with distinct spectral distribution $\mathscr{S}_{1}$ and $\mathscr{S}_{2}$ ) and three possible choices of $S_{r}\left(S_{r 1}, S_{r 2}\right.$ and $\left.S_{r 3}\right)$. The task is to select an optimal $S_{r}$ so that the discrimination between $\mathscr{S}_{1}$ and $\mathscr{S}_{2}$ is the maximum in the difference space. Referring to Figure 3 for expression in the difference space, $S_{r 1}$ is obviously a bad choice as it would have "perceived" $\mathscr{S}_{1}$ and $\mathscr{S}_{2}$ to be equidistant in the difference space. Both $S_{r 2}$ and $S_{r 3}$ lie outside the convex hull $C$ of the spectral distribution, but $S_{r 2}$ would have been a better choice as $S_{r 3}$ may risk "perceiving" $\mathscr{S}_{1}$ and $\mathscr{S}_{2}$ to be equally far in the difference space. For maximized discrimination, it is thus evident that $S_{r}$ has to chosen such that it lies just outside the convex hull of the entire spectral distribution.

\section{Metrological calculation of spectral difference}

A spectrum is essentially a continuous function $S=f(\lambda)$ defined over a spectral range $\lambda_{\min }$ to $\lambda_{\max }$ that characterizes an electromagnetic radiation [26]. Due to the sampling operation during acquisition, a spectrum is represented as a sequence of measurements $S=\left\{s_{j}(\lambda), \forall \lambda \in\left[\lambda_{\min }, \lambda_{\max }\right]\right\}$ with spectral resolution depending on the sensor. Due to its discrete representation, a spectrum is often treated as if it were a set of independent measures, hence as vectors, probability density functions or sequences [27]. This leads to the usage of L2 norms in accessing spectral difference, for instances spectral angle mapper [28] and various divergence measures $[29,30,31]$.

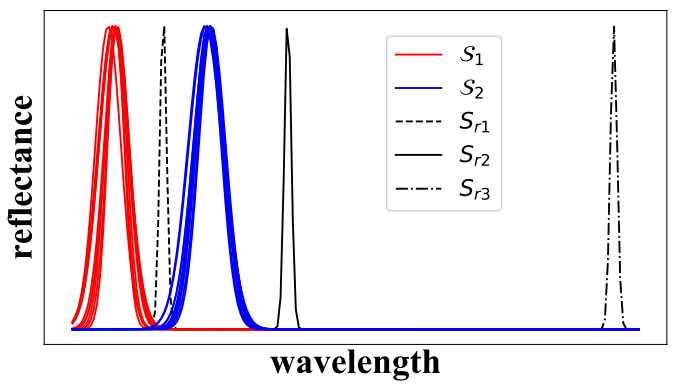

Figure 2: Representation in $P$-dimensional spectral space

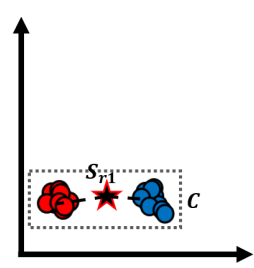

(a) $S_{r 1}$

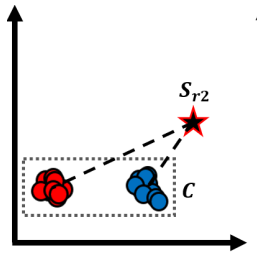

(b) $S_{r 2}$

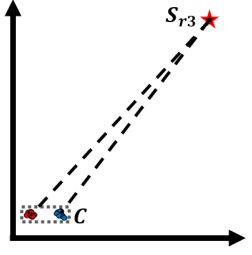

(c) $S_{r 3}$
Figure 3: Representation in $p$-dimensional difference space

However, the limits of such approach has been pointed out in [32]. While our formulation of RSDOM is not limited to any spectral difference formula, in this work we seek to express spectral difference based on the fact that spectrum is a function. As such, we use Kullback-Leibler pseudo-divergence (KLPD) [33] which is inspired from Kullback-Leibler divergence. Consider two spectra $S_{1}$ and $S_{2}$, the KLPD is defined as the sum of spectral shape difference $\Delta G$ and intensity difference $\Delta W$ as:

$$
d_{K L P D}\left(S_{1}, S_{2}\right)=\Delta G\left(S_{1}, S_{2}\right)+\Delta W\left(S_{1}, S_{2}\right)
$$

where:

$$
\begin{aligned}
& \Delta G\left(S_{1}, S_{2}\right)=k_{1} \cdot K L\left(\bar{S}_{1} \| \bar{S}_{2}\right)+k_{2} \cdot K L\left(\bar{S}_{2} \| \bar{S}_{1}\right) \\
& \Delta W\left(S_{1}, S_{2}\right)=\left(k_{1}-k_{2}\right) \log \left(\frac{k_{1}}{k_{2}}\right)
\end{aligned}
$$

as the normalized spectra $\bar{S}$ is expressed as:

$$
\bar{S}_{j}=\left\{\bar{s}_{j}(\lambda)=\frac{s_{j}(\lambda)}{k}, \forall \lambda \in\left[\lambda_{\min }, \lambda_{\max }\right]\right\}, j \in\{1,2\}
$$

with the normalization constant given by:

$$
k_{j}=\int_{\lambda_{\min }}^{\lambda_{\max }} s_{j}(\lambda) d \lambda, j \in\{1,2\}
$$

Employing KLPD, RSDOM is now a four dimensional joint probability density function given by:

$$
\begin{aligned}
& M^{(d, \theta)}\left(\Delta G, \Delta W, \Delta G_{r}, \Delta W_{r}\right)= \\
& \operatorname{Prob}\left(d_{K L P D}\left(S_{i}, S_{j}\right)=(\Delta G, \Delta W), d_{K L P D}\left(S_{i}, S_{r}\right)=\left(\Delta G_{r}, \Delta W_{r}\right)\right), \\
& \forall i, j \in H,\|\overrightarrow{i j}\|=d, \angle \overrightarrow{i j}=\theta
\end{aligned}
$$




\section{Representation using Gaussian Mixture Model}

Considering RSDOM as probability density function, fdivergence is well adapted for to access the similarity between them [34]. Among all, Kullback-Leibler (KL) divergence has been identified as the most efficient similarity measure [35]. The KL divergence [36] $K L(P \| Q)$ assess the quantity of information lost when $Q$ is used to estimate $P$ :

$$
K L(P \| Q)=\int_{-\infty}^{\infty} p(x) \ln \frac{p(x)}{q(x)} d x
$$

where $p$ and $q$ are the respective densities of $P$ and $Q$. In the particular case of multivariate normal distributions with mean $\mu$ and variance-covariance matrix $\Sigma, \mathrm{KL}$ divergence can be directly calculated by [37]:

$$
\begin{aligned}
K L(P \| Q)=\frac{1}{2}\left[\log \frac{\left|\Sigma_{Q}\right|}{\left|\Sigma_{P}\right|}+\operatorname{tr}\left(\Sigma_{Q}^{-1} \Sigma_{P}\right)-D\right. \\
\\
\left.+\left(\mu_{Q}-\mu_{P}\right)^{T} \Sigma_{Q}^{-1}\left(\mu_{Q}-\mu_{P}\right)\right]
\end{aligned}
$$

where $D$ is the dimension of data. As KL divergence is not symmetric, a similarity measure is defined as:

$$
d_{K L}(P, Q)=K L(P \| Q)+K L(Q \| P)
$$

Due to spectral variability and sensor noise, RSDOM is understandably huge and is approximately the size of the image. The direct calculation of KL divergence is therefore, computationally expensive, not to mention the huge size of RSDOM which induces problems in storage and transmission. We thus proceed to model RSDOM using Gaussian mixture model (GMM):

$$
M=\sum_{i=1}^{K} \phi_{i} \mathscr{N}\left(\mu_{i}, \Sigma_{i}\right)
$$

where $\phi$ is the mixture weight and each Gaussian component is modeled using $\mu \in \mathbb{R}^{D}$ and $\Sigma \in \mathbb{R}^{D \times D}$. Hence, RSDOM can be represented using just $K \times\left(D+D^{2}\right)$ scalar values with $D=4$ which is extremely compact.

As there is no closed form solution for KL divergence between two GMMs, we resort to using variational approximation [38] $K L_{V}$, defined as:

$$
K L_{V}\left(M \| M^{\prime}\right)=\sum_{i} \phi_{i} \log \frac{\sum_{i^{\prime}} \phi_{i^{\prime}} e^{-K L\left(M_{i} \| M_{i^{\prime}}\right)}}{\sum_{j} \phi_{j}^{\prime} e^{-K L\left(M_{i} \| M_{j}^{\prime}\right)}}
$$

as the two RSDOMs are given by $M=\sum_{i} \phi_{i} M_{i}$ and $M^{\prime}=$ $\sum_{j} \phi_{j}^{\prime} M_{j}^{\prime}$. Hence, the similarity measure is given by:

$$
d_{K L}\left(M, M^{\prime}\right)=K L_{V}\left(M \| M^{\prime}\right)+K L_{V}\left(M^{\prime} \| M\right)
$$

At this point, it is worth mentioning that as KLPD is a distance measure that takes no account in direction. This causes the distribution of RSDOM to be one-sided in the $\Delta G-\Delta W$ plane. This induces imperfection in the modeling as GMM assumes

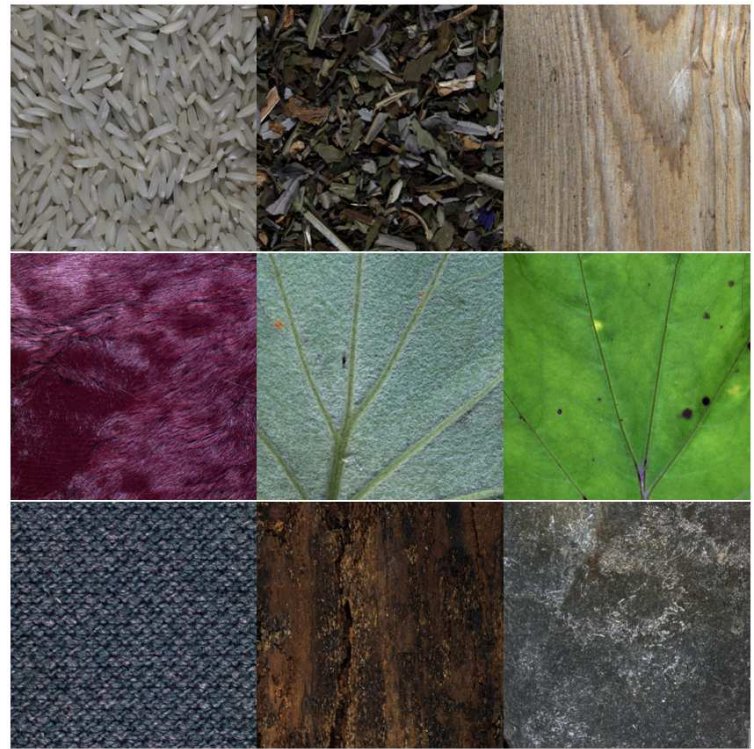

Figure 4: Several images (rendered in RGB) taken from HyTexiLa dataset

double sided distribution. Therefore, we introduce a sign operator $\operatorname{sign}(\tau)$ where $\tau$ is defined to be positive when $S_{i}$ is further from $S_{r}$ compared to $S_{j}$, or negative otherwise:

$$
\tau=d_{K L P D}\left(S_{i}, S_{r}\right)-d_{K L P D}\left(S_{j}, S_{r}\right)
$$

Hence, the final formulation of RSDOM is given by:

$$
\begin{aligned}
& M^{(d, \theta)}\left(\Delta G, \Delta W, \Delta G_{r}, \Delta W_{r}\right)= \\
& \operatorname{Prob}\left(\operatorname{sign}(\tau) \cdot d_{K L P D}\left(S_{i}, S_{j}\right)=(\Delta G, \Delta W), d_{K L P D}\left(S_{i}, S_{r}\right)=\left(\Delta G_{r}, \Delta W_{r}\right)\right), \\
& \forall i, j \in H,\|\overrightarrow{i j}\|=d, \angle \overrightarrow{i j}=\theta
\end{aligned}
$$

\section{Experiment and discussion}

To access the performance of RSDOM in hyperspectral texture recognition, we apply a classification scheme on a hyperspectral texture dataset. As comparison, we benchmark our performance against local binary pattern based approach [16]. We analyze the result and lists down the suggestions for future work

\section{The HyTexiLa dataset}

HyTexiLa (Hyperspectral Texture images acquired in Laboratory) [16] is a hyperspectral reflectance image dataset consisting of $N=112$ textured images from five categories: food (10 images), stone (4 images), textile (65 images), vegetation (15 images) and wood (18 images). The spectral count is 186 , ranging from $405.37 \mathrm{~nm}$ to $995.83 \mathrm{~nm}$ at $3.19 \mathrm{~nm}$ interval, hence spanning both visible and near infrared region. Each image measures $1024 \times 1024 \times 186$ and is acquired using the HySpex VNIR-1800 hyperspectral camera. Several images from the dataset, rendered in RGB format, are presented in Figure 4.

\section{Classification using nearest neighbor}

We perform the classification using nearest neighbor (NN) approach. In $\mathrm{NN}$, the query (test) image is compared against the training images of all classes and is assigned to the class which 


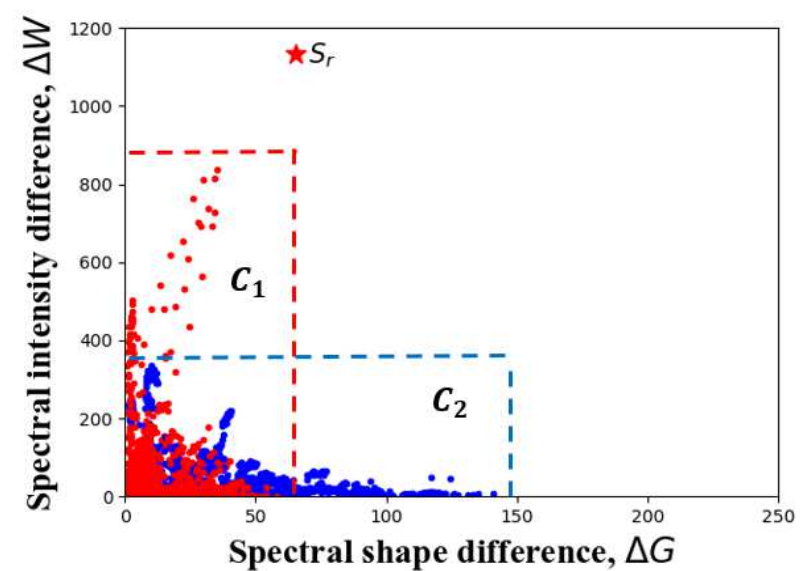

Figure 5: Choosing the reference for HyTexiLa dataset

have the highest similarity with. In the context of our classification scheme, each image, regardless of its categories, is considered a class. Following the setting in [16], each images is split into $n=25$ patches, each measuring $204 \times 204 \times 186$, of which 12 is used for training and 13 for testing. We first perform the classification on the entire dataset (denoted as "All") and later, intracategorical classifications as some category is different from others in terms of texture directionality and spectral distinctness. If the query image is classified to its own class, the classification is marked correct or otherwise false. The classification accuracy is therefore defined as the ratio of correct classification to the total number of classification. To complement the classification result, we also report the F1-score, defined as the ratio of $2($ precision $\times$ recall $)$ to $($ precision + recall $)$. To avoid any bias, we repeat all classification using 10 trials with random selection of training set and testing set in each trial. We report the average accuracy and F1-score along with standard error, with is standard deviation divided by $\sqrt{T}$ where $T$ is the number of trials.

\section{Choice of parameters}

In [16], a local binary pattern (LBP) based approach is used with $d=1$ on a neighborhood of 8 pixels. This is roughly equivalent to accessing spectral variation across three pixels. Therefore, we choose $d=3$ in the calculation of RSDOM for equal comparison. Since RSDOM is a uni-directional texture descriptor, we arbitrary choose $\theta=0$. For GMM modeling, we empirically determine that $K=4$ is optimal for RSDOM representation.

We next consider the choice of reference. Let $S_{\mu}$ be the average spectrum from each patch of $m$ pixels, defined as:

$$
S_{\mu}=\left\{s_{\mu}(\lambda)=\frac{1}{m} \sum_{i=1}^{m} s(\lambda), \forall \lambda \in\left[\lambda_{\min }, \lambda_{\max }\right]\right\}
$$

and $S_{\mu, \text { global }}$ be the global average spectrum of the entire image set containing $N$ images with $n$ patches, defined as:

$$
S_{\mu, g l o b a l}=\frac{1}{N \times n} \sum_{i=1}^{N} \sum_{j=1}^{n} S_{\mu, i, j}
$$

where $S_{\mu, i, j}$ is the average spectrum for the $j^{t h}$ patch from the $i^{t h}$ image. For simplicity, we consider $d\left(S_{\mu}, S_{\mu \text {.global }}\right)$ to obtain an rough representation of the spectral distribution for the dataset.

To choose $S_{r}$, we recall that it has to chosen such that it lies just outside the convex hull of the spectral distribution of the entire image set. However, we decide to adapt the rule considering

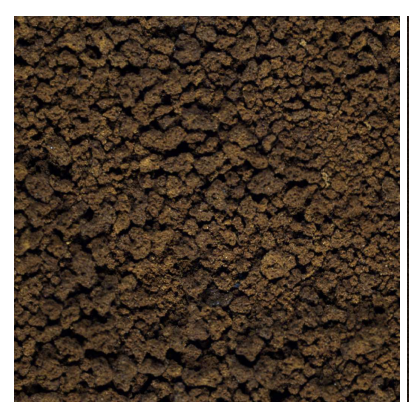

(a) coffee

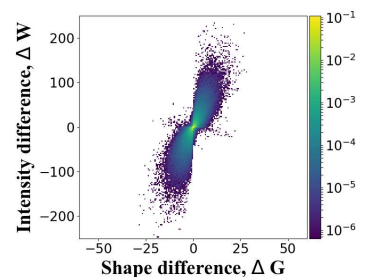

(c) RSDOM, coffee

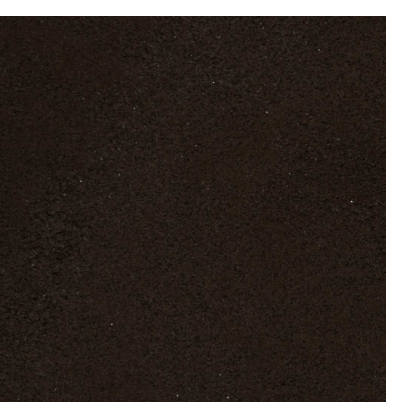

(b) milkcoffee

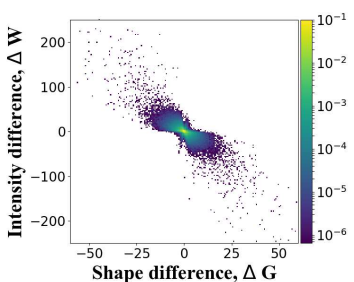

(d) RSDOM, milkcoffee
Figure 6: Representation of RSDOM for two textured images in the $\Delta W-\Delta G$ plane

the large number of images (112) with varying texture complexity between the categories. In fact, we could divide the images into two groups. Group I, enclosed by convex hull $C_{1}$, considers food, stone, vegetation and wood which are natural and complex textures. Group II, enclosed by convex hull $C_{2}$, consists of textile which are man-made and relatively simple textures. For balanced performance, we choose to focus more on the classification of Group I. Consequently, we choose $S_{r}$ such that it lies just outside $C_{1}$ as illustrated in Figure 5. By trial and error, we determine a choice of $S_{r}$ that fulfills such requirement as:

$$
S_{r}=3\left(0.5+0.5 \operatorname{erf}\left(\left(\lambda-\mu_{\lambda}\right) / \sigma_{\lambda}\right)\right)
$$

where erf is the error function. $\mu_{\lambda}$ and $\sigma_{\lambda}$ are given by:

$$
\mu_{\lambda}=\left(\lambda_{\max }-\lambda_{\min }\right) / 2
$$

$$
\sigma_{\lambda}=\left(\lambda_{\max }-\lambda_{\min }\right) / 3
$$

\section{Result and analysis}

Figure 6 illustrates RSDOM representation in the $\Delta G-\Delta W$ plane for two textured images, "coffee" and "milkcoffee". It can be seen that their spectral shape $\Delta G$ variation are similar, explainable from the fact that both "coffee" and "milkcoffee" are of the same food origin differing only on the processing level On the spectral intensity $\Delta W$, "coffee" exhibits higher variation than "milkcoffee" due to the fact that the former is coarser than the latter. This demonstrates that RSDOM is able to capture the textural properties in an easily understandable manner.

Table 1 presents the classification accuracy and F1-score using RSDOM. The classification result is excellent, with accuracy and F1-score above $90 \%$ in all cases. For the entire dataset, the 


\begin{tabular}{|c|c|c|}
\hline Category & Accuracy (\%) & F1-score (\%) \\
\hline All & $95.6 \pm 0.2$ & $95.8 \pm 0.2$ \\
\hline Food & $97.5 \pm 0.4$ & $97.5 \pm 0.3$ \\
\hline Stone & $98.3 \pm 0.5$ & $98.3 \pm 0.5$ \\
\hline Vegetation & $93.7 \pm 0.5$ & $94.1 \pm 0.5$ \\
\hline Wood & $90.8 \pm 0.7$ & $91.2 \pm 0.7$ \\
\hline Textile & $98.6 \pm 0.1$ & $98.7 \pm 0.1$ \\
\hline
\end{tabular}

Table 1: Classification accuracy and F1-score on HyTexiLa database

classification accuracy reaches $95.6 \%$. To further analyze this result, we refer to the normalized confusion matrix as shown in Figure 7. As there are too many class (112 in total) which would rendered examination difficult, we display the confusion matrix in two parts. Figure 7(a) shows the confusion matrix with textile (T) classes clustered together, while Figure 7(b) shows the confusion matrix with food $(\mathrm{F})$, stone $(\mathrm{S})$, vegetation $(\mathrm{V})$ and wood (W) classes clustered together. It is clear that the misclassification is largely due to two categories: wood and vegetation. This is also supported from the intra-categorical classification result in Table 1 which shows their lower accuracy compared to others.

We highlight three reasons to explain the misclassification. Firstly, the texture are mainly green for vegetation and brown for wood. Therefore, the makes discrimination within the categories more difficult. Secondly, our unitary choice of $d=3$ prevents us from assessing texture with varying texton [39] size. This explains our higher score in food, stone and textile which consist of uniform and fine textons, but lower score in wood and vegetation which has varying texton size. Thirdly, our unitary choice of $\theta$ allows us to only evaluate texture in one direction. In fact, for $\theta=0$ we are only able to assess texture varying in vertical direction but not in other directions. This is acceptable for isotropic texture, but not adapted for anisotropic texture such as those found in vegetation and wood categories.

In comparison to [16] which reports an accuracy of 98.76 $\%$ using Opponent Band Local Binary Pattern (OBLBP), our performance is lower although in the same efficiency range. The difference in performance can be attributed to the fact that OBLBP assesses texture in eight directions while RSDOM evaluates just one. However, it is worthwhile to mention that the feature size of RSDOM is extremely small. While OBLBP consists of 82944 scalar values, the size of RSDOM is only $K \times\left(D+D^{2}\right)=80$ scalar values or $0.1 \%$ of OBLBP's. Even if RSDOM is adapted to assess texture in eight directions as in the case of OBLBP, its feature size only increases to $8 \times K \times\left(D+D^{2}\right)=640$ scalar values. This presents RSDOM as an extremely compact feature descriptor for rapid processing and storage.

\section{Limitations and future work}

The choice of $d=3$ remain questionable, as it is possibly susceptible to image noise and it is expected that a larger $d$ value could present better result. In fact, as texture varies in scale and direction, RSDOM could benefit from a multi-scale and multidirection implementation. On the other hand, the choice of using variational approximation in this work to evaluate divergence between two RSDOM which are GMMs remains to be justified. Other methods ought to be studied in order to determine the best distance measure. ast but no least, other approaches of modeling RSDOM such as Generalized Gaussian Distribution (GGD) or shape descriptions (Fourier Descriptor, Zernike moments, Curvature Scale Space etc.) are also worth being studied.

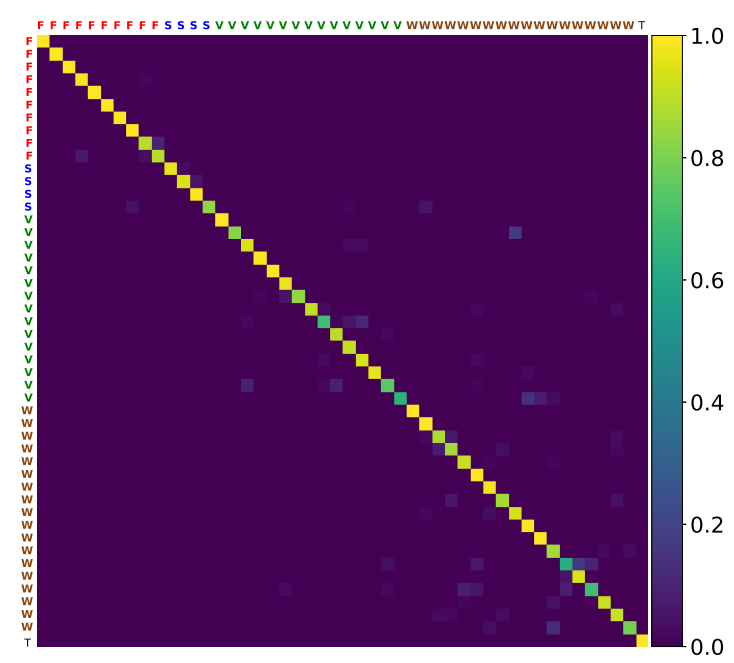

(a) Textile classes clustered togteher

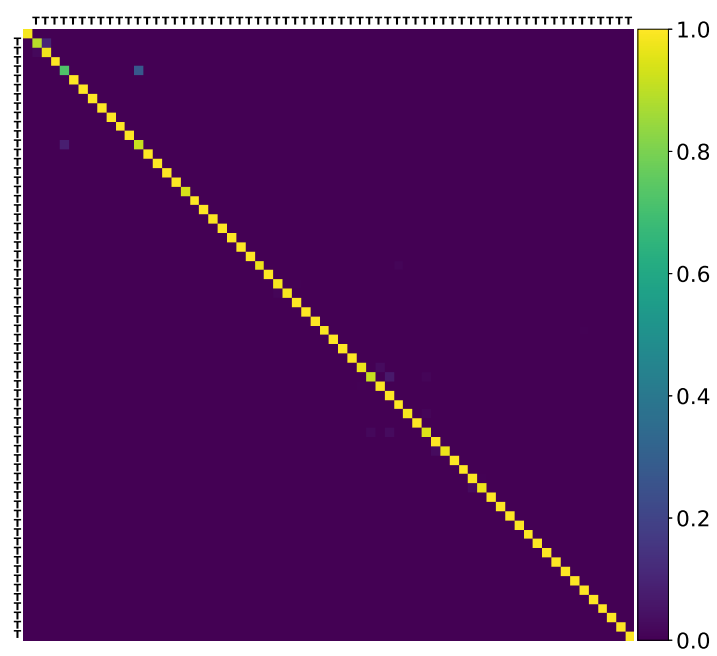

(b) Food, stone, vegetation and wood classes clustered togteher

Figure 7: Normalized confusion matrix for HyTexiLa classification ( $\mathrm{F}=$ food, $\mathrm{S}=$ stone, $\mathrm{V}=$ vegetation, $\mathrm{W}=$ wood, $\mathrm{T}=$ textile)

\section{Conclusion}

We have proposed a spectral-spatial feature, Relative Spectral Difference Occurrence Matrix (RSDOM) for hyperspectral texture recognition. The proposed feature is in full accordance with psychophysical experiments on texture. It simultaneously considers the distribution of spectra and their spatial arrangement in the hyperspectral image. It is generic and adapted for any number of spectral bands and range. Thanks to its operation in the difference space, the curse of dimensionality is overcome without requiring band selection or dimensionality reduction techniques, therefore preserving the metrological properties. Its performance has been evaluated by applying a classification scheme on the HyTexiLa dataset with excellent accuracy (95.6\%). The result is comparable to Local Binary Pattern (LBP) based approaches, but at a much smaller feature size ( $0.1 \%$ of the LBP-based feature). 


\section{References}

[1] K. Masood and N. Rajpoot, Texture based classification of hyperspectral colon biopsy samples using CLBP, 2009 IEEE International Symposium on Biomedical Imaging: From Nano to Macro, pp 1011-1014 (2009).

[2] R. Awan, S. Al-Maadeed and R. Al-Saady, Using spectral imaging for the analysis of abnormalities for colorectal cancer: When is it helpful?, PLoS One, 13, 6 (2018).

[3] L. Liu, M. O. Ngadi, S. O. Prasher and C. Gariepy, Categorization of pork quality using Gabor filter-based hyperspectral imaging technology, Journal of Food Engineering, 99, 3 (2010).

[4] Y. Feng and D. Sun, Application of Hyperspectral Imaging in Food Safety Inspection and Control: A Review, Critical Reviews in Food Science and Nutrition, 52, 11 (2012).

[5] G. Rellier, X. Descombes, F. Falzon and J. Zerubia, Texture feature analysis using a gauss-Markov model in hyperspectral image classification, IEEE Transactions on Geoscience and Remote Sensing, 42, 7 (2004).

[6] J. Zhang, W. Geng, X. Liang, J. Li, L. Zhuo and Q. Zhou, Hyperspectral remote sensing image retrieval system using spectral and texture features, Applied Optics, 56, 16 (2017).

[7] F. Tsai and J. Lai, Feature Extraction of Hyperspectral Image Cubes Using Three-Dimensional Gray-Level Cooccurrence, IEEE Transactions on Geoscience and Remote Sensing, 51, 6 (2013).

[8] S. Jia, J. Hu, J. Zhu, X. Jia and Q. Li, Three-Dimensional Local Binary Patterns for Hyperspectral Imagery Classification, IEEE Transactions on Geoscience and Remote Sensing, 55, 4 (2017).

[9] X. Kang, C. Li, S. Li and H. Lin, Classification of Hyperspectral Images by Gabor Filtering Based Deep Network, IEEE Journal of Selected Topics in Applied Earth Observations and Remote Sensing, 11, 4 (2018)

[10] S. L. Moan, A. Mansouri, Y. Voisin and J. Y. Hardeberg, A constrained band selection method based on information measures for spectral image color visualization, IEEE Transactions on Geoscience and Remote Sensing, 49, 12 (2011).

[11] B. Guo, S. R. Gunn, R. I. Damper and J. D. B. Nelson, Band Selection for Hyperspectral Image Classification Using Mutual Information, IEEE Geoscience and Remote Sensing Letters, 3, 4 (2006).

[12] R. Yang, L. Su, X. Zhao, H. Wan and J. Sun, Representative band selection for hyperspectral image classification, Journal of Visual Communication and Image Representation, 48 (2017).

[13] H. Zeng and H. J. Trussell, Dimensionality reduction in hyperspectral image classification, 2004 International Conference on Image Processing, pp. 913-916 (2004).

[14] J. Khodr and R. Younes, Dimensionality reduction on hyperspectral images: A comparative review based on artificial datas, 2011 4th International Congress on Image and Signal Processing, pp. 18751883 (2011).

[15] H. Huang and M. Yang, Dimensionality Reduction of Hyperspectral Images With Sparse Discriminant Embedding, IEEE Transactions on Geoscience and Remote Sensing, 53, 9 (2015).

[16] H. A. Khan, S. Mihoubi, B. Mathon, J. B. Thomas and J. Y. Hardeberg, Hytexila: High resolution visible and near infrared hyperspectral texture images, Sensors, 18, 7 (2018).

[17] S. Wang and C. Wang, Research on dimension reduction method for hyperspectral remote sensing image based on global mixture coordination factor analysis, ISPRS - International Archives of the Photogrammetry, Remote Sensing and Spatial Information Sciences, pp. 159-167 (2015).

[18] P. DeBievre, The 2012 International Vocabulary of Metrology: "VIM", Accreditation and Quality Assurance, 17, 2 (2012).

[19] B. Julesz, Visual pattern discrimination, Transactions on Information Theory, 8, 2 (1962)
[20] B. Julesz, E. N. Gilbert, L. A. Shepp and H. L. Frisch, Inability of Humans to Discriminate between Visual Textures That Agree in Second-Order Statistics-Revisited, Perception, 2, 4 (1973).

[21] R. M. Haralick, K. Shanmugam, and I. Dinstein, Textural feature for image classification, IEEE Transactions on Systems, Man and Cybernetics, 3, 6 (1973).

[22] T. Ojala, M. Pietikainen, and T. Maenpaa, Multiresolution grayscale and rotation invariant texture classification with local binary patterns, IEEE Transactions on Pattern Analysis and Machine Intelligence, 24, 7 (2002).

[23] M. Unser, Sum and difference histograms for texture classification, IEEE Transactions on Pattern Analysis and Machine Intelligence, 8, 1 (1986).

[24] R. W. Ehrich and J. P. Foith, A view of texture topology and texture description, Computer Graphics and Image Processing, 8, 2 (1978).

[25] H. Deborah, Towards Spectral Mathematical Morphology, Norwegian University of Science and Technology, University of Poitiers, 2016, pp. 113-116.

[26] H. Fritzsche and M. Phillips, Electromagnetic radiation, Encyclopedia Britannica, Encyclopedia Britannica Inc., October 18, 2017, https://www.britannica.com/science/electromagneticradiation/Continuous-spectra-of-electromagnetic-radiation

[27] H. Deborah, N. Richard and J. Y. Hardeberg, A comprehensive evaluation on spectral distance functions and metrics for hyperspectral image processing, IEEE Journal of Selected Topics in Applied Earth Observations and Remote Sensing, 8, 6 (2015).

[28] R. H. Yuhas, A. F. H. Goetz and J. W. Boardman, Discrimination among semi-arid landscape endmembers using the Spectral Angle Mapper (SAM) algorithm, JPL, Summaries of the Third Annual JPL Airborne Geoscience Workshop. Volume 1: AVIRIS Workshop, pp. 147-149 (1992)

[29] H. Jeffreys, An invariant form for the prior probability in estimation problems, Proceedings of the Royal Society of London. Series A. Mathematical and Physical Sciences, pp. 453-461 (1946).

[30] Y. Rubner, Perceptual metrics for image database navigation, Stanford University, Stanford, CA, 1999.

[31] Y. Rubner, C. Tomasi and L. J. Guibas, The Earth Mover's Distance as a Metric for Image Retrieval, International Journal of Computer Vision, pp. 99-121 (2000).

[32] N. Richard, H. Deborah and O. Ahmed, Hyperspectral image processing: Full-band, dimensionality reduction, or band selection approach?, Treatment and Analysis of the Information Methods and Applications (TAIMA) (2018)

[33] N. Richard, D. Helbert, C. Olivier, and M. Tamisier, Pseudodivergence and bidimensional histogram of spectral differences for hyperspectral image processing, Journal of Imaging Science and Technology, 60, 5 (2016).

[34] M. Basseville, Distance measures for signal processing and pattern recognition, Signal Processing, 18, 4 (1989).

[35] Z. Bylinskii, T. Judd, A. Oliva, A. Torralba and F. Durand, What do different evaluation metrics tell us about saliency models?, IEEE Transactions on Pattern Analysis and Machine Intelligence, 41, 3 (2019).

[36] S. Kullback and R. A. Leibler, On information and sufficiency, The Annals of Mathematical Statistics, 22, 1 (1951).

[37] C. E. Rasmussen and C. K. I. Williams, Gaussian Processes for Machine Learning, MIT Press, Massachusetts, MA, 2006, p. 203.

[38] J. R. Hershey and P. A. Olsen, Approximating the Kullback Leibler Divergence Between Gaussian Mixture Models, 2007 IEEE International Conference on Acoustics, Speech and Signal Processing ICASSP '07, pp. 317-320 (2007).

[39] B. Julesz, Textons, the elements of texture perception, and their interactions, Nature, 290 (1981) 


\section{Author Biography}

Rui Jian Chu is a doctoral researcher at University of Poitiers. He obtained his BE (Hons) in electrical and computer systems from Monash University in 2016. Then, he received his MSc. from Jean Monnet University, University of Granada and University of Eastern Finland, all in 2018. Since 2019, he is conducting PhD research concerning texture features for hyperspectral image analysis. He is particularly interested in developing metrological approaches to address spatio-chromatic complexity in digital images. 